\title{
Neutrophil-to-lymphocyte ratio is associated with sensitivity to platinum-based chemotherapy and prognosis in patients with advanced serous ovarian carcinoma
}

\author{
TAKESHI FUKUDA ${ }^{1}$, MASARU KAWANISHI ${ }^{1,2}$, YUICHIRO AWAZU ${ }^{1}$, SHIGENORI NANNO $^{1}$, \\ MASAHIRO SHIMOMURA ${ }^{1}$, YUTA INOUE ${ }^{1}$, HIROAKI MATSUBARA ${ }^{1}$, MAKOTO YAMAUCHI $^{1}$, MARI KASAI ${ }^{1}$, \\ YASUNORI HASHIGUCHI ${ }^{1}$, TOMOYUKI ICHIMURA ${ }^{1}$, TOMOYO YASUI ${ }^{1}$ and TOSHIYUKI SUMI $^{1}$ \\ ${ }^{1}$ Department of Obstetrics and Gynecology, Osaka City University Graduate School of Medicine, Osaka 545-8585; \\ ${ }^{2}$ Department of Gynecology, Osaka City General Hospital, Osaka 534-0021, Japan
}

Received March 10, 2021; Accepted May 11, 2021

DOI: $10.3892 / \mathrm{mco} .2021 .2381$

\begin{abstract}
The role of the neutrophil-to-lymphocyte ratio (NLR) in predicting sensitivity to chemotherapy and prognosis has attracted great interest in several types of cancer. In the present study, the correlation between pre-chemotherapy NLR and sensitivity to platinum-based chemotherapy and prognosis in patients with advanced serous ovarian carcinoma was examined by retrospectively reviewing the medical records of 50 patients with stage III-IV serous ovarian carcinoma from 2005 to 2012. Patients were divided into high-NLR (32 patients) and low-NLR (18 patients) groups according to a cutoff value of 2.47. This cutoff was calculated using a receiver operating characteristic (ROC) curve that demonstrated $84 \%$ specificity and $60 \%$ sensitivity. Patient characteristics, sensitivity to platinum-based chemotherapy and prognosis were subsequently compared. The results revealed no significant difference in patient characteristics between the two groups. In the low-NLR group, 14 of 18 patients (77.8\%) were sensitive to platinum-based chemotherapy, whereas 11 of 32 were sensitive in the high-NLR group (34.4\%) $(\mathrm{P}=0.007)$. Overall and disease-free survival (DFS) were significantly longer in the low-NLR than in the high-NLR group $(\mathrm{P}=0.013$ and $\mathrm{P}=0.043$, respectively). The current results suggested that pre-chemotherapeutical NLR may serve as a biomarker of sensitivity to platinum-based chemotherapy and prognosis in patients with advanced serous ovarian carcinoma.
\end{abstract}

Correspondence to: Dr Takeshi Fukuda, Department of Obstetrics and Gynecology, Osaka City University Graduate School of Medicine, 1-4-3 Asahimachi, Abeno-ku, Osaka 545-8585, Japan E-mail: takeshif@med.osaka-cu.ac.jp

Key words: ovarian serous carcinoma, neutrophil-to-lymphocyte ratio, chemotherapy, predictive marker, chemosensitivity, prognosis

\section{Introduction}

Epithelial ovarian carcinoma is a common cause of cancer deaths in women worldwide. The predominant subtype is serous ovarian carcinoma, which accounts for more than $50 \%$ of all ovarian carcinomas (1). The diagnosis is generally made at an advanced stage because of the insidious disease onset and lack of effective screening strategies. The standard treatment for these patients consists of primary debulking surgery followed by platinum-based chemotherapy. The initial response to first-line platinum-based chemotherapy is favorable; however, most patients develop recurrences and resistance to platinum-based chemotherapy, resulting in poor 5- and 10-year survivals of $\sim 32$ and $15 \%$ respectively $(1,2)$. There are currently no biomarkers that reliably predict the response to platinum-based chemotherapy. Identification of dependable biomarkers is needed to facilitate planning optimal personalized treatment strategies and predicting the prognosis of patients with advanced serous ovarian carcinoma.

Systemic inflammation plays a crucial role in the development and progression of several types of cancer. Systemic inflammation can up-regulate cytokines and inflammatory mediators, inhibit apoptosis, initiate angiogenesis, remodel the extracellular matrix, and trigger DNA damage $(3,4)$. Biological indicators of the severity of systemic inflammation include C-reactive protein, neutrophil-to-lymphocyte ratio (NLR), platelet-to-lymphocyte ratio, lymphocyte-to-monocyte ratio, and platelet count (5-9). Among these indicators, NLR has been attracting great interest because it is reproducible and easy and inexpensive to measure in routine clinical practice. Several researchers have reported the association between a high NLR and poor prognosis in patients with various types of carcinoma (9-12). Furthermore, a high NLR is reportedly a useful predictor of poor response to treatment and disease recurrence (13-15).

Several studies into the relationship between NLR and prognosis of ovarian carcinoma have been published; however, their conclusions are controversial. Most of these studies have focused on pre-treatment NLRs. We evaluated the relationship between NLR before initiating chemotherapy, 
after primary debulking surgery, and sensitivity to platinum-based chemotherapy and prognosis. We considered that the pre-chemotherapy NLR would more accurately reflect the severity of inflammation and predict the sensitivity to chemotherapy than the pre-surgery NLR. To the best of our knowledge, this is the first reported investigation of this hypothesis.

\section{Materials and methods}

Patients and data. This study was a retrospective study of data drawn from patients' medical records. We reviewed the records of 50 patients with stage III or IV serous ovarian cancer treated at Osaka City University Hospital between January 2005 and December 2012. High-grade serous ovarian carcinoma had been diagnosed histologically in all patients. We excluded patients for whom pre-treatment neutrophil or lymphocyte counts were unavailable. All patients had undergone primary debulking surgery followed by six 3-weekly cycles of carboplatin plus paclitaxel. We collected information on the following clinical variables: Age, Federation of Gynaecology and Obstetrics (FIGO) stage, serum cancer antigen (CA125) concentration, size of postoperative residual tumor, leukocyte count, and response to platinum-based chemotherapy. The NLR was defined as the neutrophil count divided by the lymphocyte count and was calculated 2 days before initiation of chemotherapy, which was commenced $\sim 2$ weeks after primary debulking surgery. A ROC curve was generated to determine the cutoff value of NLR for predicting sensitivity to platinum-based chemotherapy. Patients were allocated to low-NLR group and high-NLR group on the basis of this cutoff value.

We obtained informed consent for treatment from all patients and received approval from the Institutional Review Board of Osaka City University Hospital before initiating this study (IRB no. 2020-288).

Chemotherapy and evaluation of the effect of treatment. All patients underwent six 3-weekly cycles of chemotherapy with paclitaxel plus carboplatin $\left(175 \mathrm{mg} / \mathrm{m}^{2}\right.$ of paclitaxel infused $>3 \mathrm{~h}$; dosage of carboplatin calculated with an area under the curve of 5 and infused $>1 \mathrm{~h}$ ). Platinum resistance was defined as $<6$ months between last dose of platinum and recurrence and platinum sensitivity as longer than 6 months between last platinum dose and recurrence. DFS was defined as the time between dates of diagnosis and disease recurrence. Overall survival (OS) was defined as the time between dates of diagnosis and death or most recent follow-up.

Statistical analysis. We compared characteristics, sensitivity to platinum-based chemotherapy, OS, and DFS between patients in the low-NLR and high-NLR groups. EZR software version 1.3 (Saitama Medical Center, Jichi Medical University, Saitama, Japan) was used for all statistical analyses. The data are represented as mean \pm standard deviation. Student's t-test was used to compare differences between the data. Fisher's exact test was used to identify differences in the distribution of categorical variables between groups. Kaplan-Meier plots and log-rank tests were used to analyze OS and DFS. P-value $<0.05$ was considered to denote statistical significance.
Table I. Patient characteristics.

\begin{tabular}{lc}
\hline Characteristic & Value \\
\hline Number of patients & 50 \\
Age, years & \\
Mean \pm SD & $60.64 \pm 10.89$ \\
Range & $36-79$ \\
FIGO stage, $\mathrm{n}(\%)$ & \\
IIIA & $1(2)$ \\
IIIB & $4(8)$ \\
IIIC & $37(74)$ \\
IVA & $5(10)$ \\
IVB & $3(6)$ \\
CA125 (U/ml) & \\
Mean \pm SD & $2365.3 \pm 3063.9$ \\
Range & $62-12,300$ \\
NLR before chemotherapy & \\
Mean \pm SD & $4.04 \pm 3.83$ \\
Range & $0.89-18.8$ \\
Postoperative residual tumor, $\mathrm{n}(\%)$ & \\
None & $5(10)$ \\
$\leq 1 \mathrm{~cm}$ & $12(24)$ \\
$>1 \mathrm{~cm}$ & $33(66)$ \\
Sensitivity to platinum-based & \\
chemotherapy, $\mathrm{n}(\%)$ & \\
Sensitive & $25(50)$ \\
Resistant & $25(50)$ \\
\hline
\end{tabular}

SD, standard deviation; FIGO, International Federation of Gynecology and Obstetrics; NLR, neutrophil-to-lymphocyte ratio.

\section{Results}

Patient characteristics. The characteristics of all patients are shown in Table I. Their age ranged from 36 to 79 years (mean age, 60.64 years). The most prevalent disease stage was IIIC, accounting for $74 \%$ of all patients. Pre-chemotherapy NLRs ranged from 0.89 to 18.8 (mean, 4.04). Half of the patients were sensitive to platinum-based chemotherapy and the other resistant to it.

ROC curve for determining NLR cutoff value for predicting sensitivity to platinum-based chemotherapy. We generated an ROC curve to determine the cutoff value for predicting sensitivity to platinum-based chemotherapy (Fig. 1). This resulted in a cutoff value of 2.47 . The specificity for prediction of sensitivity to platinum-based chemotherapy was $84 \%$ and the sensitivity of this cutoff value was $60 \%$. We therefore adopted an NLR cutoff of 2.47 for allocating patients to high-NLR ( $\geq 2.47)$ and low-NLR (<2.47) groups.

Characteristics of high- and low-NLR groups and comparison of platinum sensitivity between group. Table II shows patient characteristics according to groups. We compared 
Table II. Characteristics of patients in the low- and high-NLR groups.

\begin{tabular}{|c|c|c|c|}
\hline Characteristic & Low-NLR group $(<2.47)$ & High-NLR group ( $\geq 2.47$ ) & P-value \\
\hline No. of patients & 18 & 32 & - \\
\hline Age, years & & & $0.821^{\mathrm{a}}$ \\
\hline Mean \pm SD & $61.11 \pm 9.76$ & $60.38 \pm 11.62$ & \\
\hline Range & $47-75$ & $36-79$ & \\
\hline FIGO stage, $\mathrm{n}$ & & & $0.588^{\mathrm{b}}$ \\
\hline IIIA & 0 & 1 & \\
\hline IIIB & 1 & 3 & \\
\hline IIIC & 16 & 21 & \\
\hline IVA & 1 & 4 & \\
\hline IVB & 0 & 3 & \\
\hline CA125 (U/ml) & & & $0.641^{\mathrm{a}}$ \\
\hline Mean \pm SD & $2,638.11 \pm 3,416.06$ & $2,211.88 \pm 2,876.52$ & \\
\hline Postoperative residual tumor, $\mathrm{n}$ & & & $0.115^{\mathrm{b}}$ \\
\hline None & 4 & 1 & \\
\hline$\leq 1 \mathrm{~cm}$ & 4 & 8 & \\
\hline$>1 \mathrm{~cm}$ & 10 & 23 & \\
\hline
\end{tabular}

Significance was determined using a ${ }^{a}$ Student t-test or ${ }^{b}$ Fisher's exact test. NLR, neutrophil-to-lymphocyte ratio; FIGO, International Federation of Gynecology and Obstetrics; SD, standard deviation.

Table III. Number of platinum-sensitive and platinum-resistant patients in the low- and high-NLR groups.

\begin{tabular}{|c|c|c|c|}
\hline Variable & Low-NLR group $(\leq 2.47) \mathrm{n}=18$ & High-NLR $(\geq 2.47) n=32$ & P-value \\
\hline Platinum-sensitive, n (\%) & $14(77.8)$ & $11(34.4)$ & $0.007^{\mathrm{a}}$ \\
\hline Platinum-resistant, n (\%) & $4(22.2)$ & $21(65.6)$ & \\
\hline
\end{tabular}

Significance was determined using a a Fisher's exact test. NLR, neutrophil-to-lymphocyte ratio.

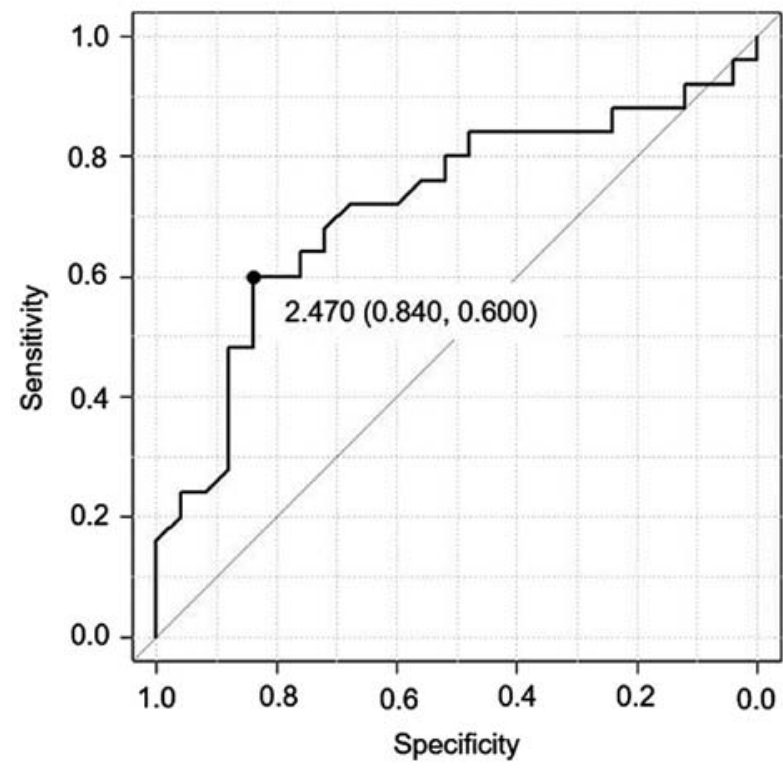

Figure 1. Receiver operating characteristic curve determining the cutoff value of neutrophil-to-lymphocyte ratio for the prediction of sensitivity to platinum-based chemotherapy. Area under the curve $=0.717 ; 95 \%$ confidence interval, $0.568-0.866$. age, FIGO stage, serum CA125 concentration, and size of postoperative residual tumor. None of the studied factors differed significantly between the groups, suggesting that the NLR value was the only difference between them. Next, we compared sensitivity to platinum-based chemotherapy between the two groups. Table III shows the number of patients with platinum-sensitive and platinum-resistant disease in each group. In the low-NLR group, $77.8 \%$ of patients were sensitive to platinum-based chemotherapy, whereas in the high-NLR group $34.4 \%$ were sensitive to it. Thus, the low-NLR group was significantly more sensitive to platinum-based chemotherapy than was the high-NLR group $(\mathrm{P}=0.007)$.

Comparison of prognosis between high-and low-NLR groups. Fig. 2 shows the OS and DFS of the two groups. The low-NLR group had significantly better OS and DFS than did the high-NLR group ( $\mathrm{P}=0.013$ and $\mathrm{P}=0.043$, respectively). These results suggest that the NLR can serve as a biomarker for predicting the prognosis of patients with serous ovarian carcinoma who undergo debulking surgery followed by platinum-based chemotherapy. 
A

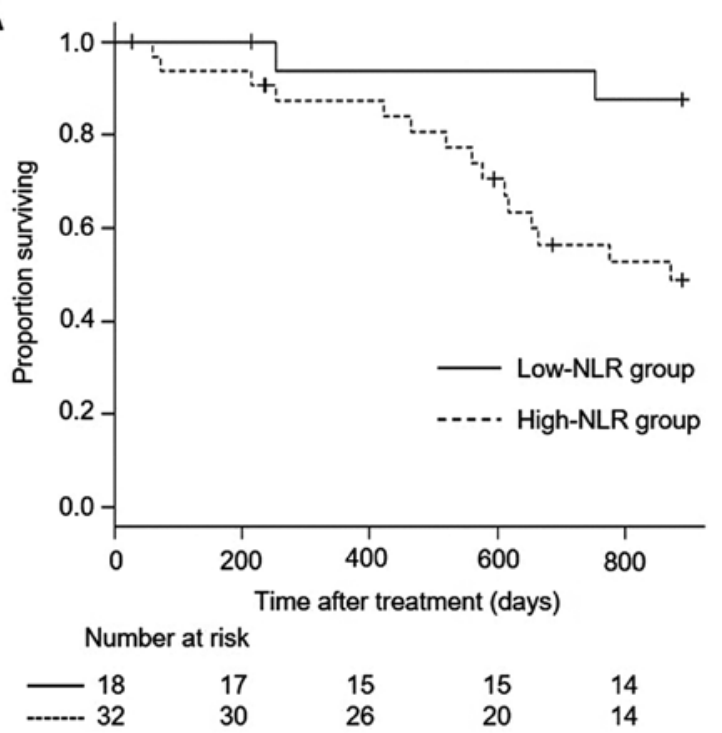

B

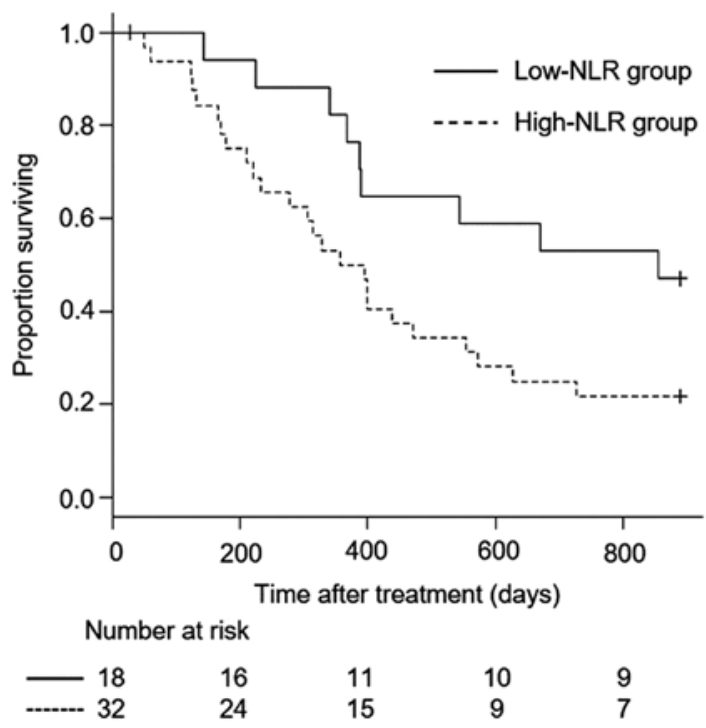

Figure 2.Overall and disease-free survival according to neutrophil-to-lymphocyte ratios (NLR). (A) Overall survival ( $\mathrm{P}=0.013$ ). (B) Disease-free survival $(\mathrm{P}=0.043)$.

\section{Discussion}

In this study of patients with ovarian serous carcinoma who had undergone debulking surgery followed by platinum-based chemotherapy, we investigated the ability of pre-chemotherapy NLR to predict sensitivity to platinum-based chemotherapy and prognosis. We found that the pre-chemotherapy NLR predicted sensitivity to chemotherapy and prognosis when we used a cutoff value of 2.47 .

The crucial impact of inflammation and the associated leukocyte recruitment on cancer development was first reported in 1863 by Virchow. It is now clear that inflammation-related neutrophils and immunocytes are components of the tumor microenvironment and play an essential role in the neoplastic process by fostering proliferation of, and communication between cancer cells and that microenvironment $(3,16,17)$. Tumor development and progression are initiated via DNA damage and overproduction of cytokines such as vascular endothelial growth factor (VEGF), tumor necrosis factor alpha (TNF- $\alpha$ ), and interleukin (IL)-2 and IL-6 $(16,18)$. The inflammatory response involves clustering of immune cells, including tumor-associated macrophages and neutrophils, tumor-induced T cells, dendritic cells, and innate lymphoid cells. This accumulation of cells prompts initiation and progression of cancer and may simultaneously suppress cancer progression (19-22).

Neutrophils account for 50-60\% of leukocytes and become more numerous in the presence of inflammation. An increase in neutrophil count indicates systemic inflammation and has been reported to be involved in tumor proliferation, invasion, and angiogenesis $(3,23,24)$. Cancer-associated angiogenesis and cellular DNA damage are promoted by cytotoxic mediators such as reactive oxygen species and neutrophil elastase, which are released by neutrophils (25). Neutrophils recruit inflammatory mediators, including IL-1, IL-6, TNF, and VEGF, and then inhibit the cytotoxic activity of lymphocytes, impairing activation of adaptive immunity $(26,27)$. Neutrophils can facilitate tumor development by remodeling the extracellular matrix, providing pro-angiogenic factors, and inhibiting lymphocyte activity (28).

Hematological markers that reflect systemic inflammation, such as the NLR, have recently attracted considerable interest as predictors of prognosis and sensitivity to chemotherapy in patients with cancer. An increasing number of researchers have reported a relationship between pre-operative NLR and prognosis in patients with ovarian cancer. However, several studies have failed to confirm this relationship (29). Additionally, the mechanism(s) by which a high NLR contributes to poor prognosis and poor sensitivity to chemotherapy is still poorly understood. The NLR precisely expresses the balance between neutrophils and immunocytes, a high NLR reflecting an up-regulated innate immune response with increased concentrations of cytokines and tumor macrophage infiltration. The NLR has therefore been considered a novel prognostic indicator $(30,31)$. It is also reportedly associated with the responses of several cancers to chemotherapy (32-35), indicating it potentially has value as a biomarker for predicting sensitivity to chemotherapy. Blood cell counts are routinely checked at the beginning of diagnosis or treatment, making the NLR a convenient, cost-effective, and reproducible marker in clinical practice. However, varying means of determining optimal thresholds have been used to predict prognosis and sensitivity to chemotherapy. Some studies have used ROC analysis to calculate the threshold, others have used the median NLR, and still other have used the interquartile limits. The resultant lack of consensus on threshold values, and therefore on definitions of normal and high NLRs, has hindered the use of the NLR both in daily clinical practice and in investigating the relationship between NLR and prognosis in patients with ovarian cancer.

To the best of our knowledge, this is the first study to investigate the relationships between pre-chemotherapeutic NLR and sensitivity to platinum-based chemotherapy and prognosis in patients with serous ovarian carcinoma. In this study, we used the post-debulking surgery, pre-chemotherapy NLR rather than the NLR before initiation of any treatment because we believe that the former more accurately reflects 
the status of cancer-related inflammation and therefore more reliably predicts tumor sensitivity to chemotherapy. We found that pre-chemotherapy NLR is associated with sensitivity to platinum-based chemotherapy and prognosis in patients with advanced high-grade serous ovarian carcinoma. Several studies have used the pre-treatment NLR to predict the sensitivity to chemotherapy or prognosis. However, to the best of our knowledge, no studies have investigated the post-debulking surgery, pre-chemotherapy NLR.

The limitations of this study include that it was a relatively small, single institution, retrospective study using univariate analysis. Further investigation, including validation in a second cohort, are needed before NLR can be confidently recommended in clinical practice. However, we believe that our findings contribute to progress in the use of the NLR to predict sensitivity to platinum-based chemotherapy and prognosis in patients with serous ovarian carcinoma.

\section{Acknowledgements}

Not applicable.

\section{Funding}

This study was funded by The Osaka Medical Research Foundation for Intractable Diseases (grant no. 26-2-47).

\section{Availability of data and materials}

The datasets used and/or analyzed during the present study are available from the corresponding author on reasonable request.

\section{Authors' contributions}

TF and TS designed the present study. TF, MKaw, YA, SN, MS, YI, HM, MY, MKas, YH, TI and TY collected and analyzed the data. TF, MKaw and TS wrote the manuscript. TF and MKaw confirm the authenticity of all the raw data. All authors read and approved the final manuscript.

\section{Ethics approval and consent to participate}

The present study was approved by the Institutional Review Board of Osaka City University Hospital before its initiation (approval no. 2020-288). Written informed consent for all treatment was obtained from all patients.

\section{Patient consent for publication}

Not applicable.

\section{Competing interests}

The authors declare that they have no competing interests.

\section{References}

1. Peres LC, Cushing-Haugen KL, Köbel M, Harris HR, Berchuck A Rossing MA, Schildkraut JM and Doherty JA: Invasive epithelial ovarian cancer survival by histotype and disease stage. J Natl Cancer Inst 111: 60-68, 2019.
2. Cannistra SA: Cancer of the ovary. N Engl J Med 351: 2519-2529, 2004.

3. Coussens LM and Werb Z: Inflammation and cancer. Nature 420 : 860-867, 2002

4. Hanahan D and Weinberg RA: Hallmarks of cancer: The next generation. Cell 144: 646-674, 2011.

5. Buergy D, Wenz F, Groden C and Brockmann MA: Tumor-platelet interaction in solid tumors. Int J Cancer 130: 2747-2760, 2012.

6. Nishijima TF, Muss HB, Shachar SS, Tamura K and Takamatsu Y: Prognostic value of lymphocyte-to-monocyte ratio in patients with solid tumors: A systematic review and meta-analysis. Cancer Treat Rev 41: 971-978, 2015.

7. Shrotriya S, Walsh D, Bennani-Baiti N, Thomas S and Lorton C: C-reactive protein is an important biomarker for prognosis tumor recurrence and treatment response in adult solid tumors: A systematic review. PLoS One 10: e0143080, 2015.

8. Templeton AJ, Ace O, McNamara MG, Al-Mubarak M, Vera-Badillo FE, Hermanns T, Seruga B, Ocaña A, Tannock IF and Amir E: Prognostic role of platelet to lymphocyte ratio in solid tumors: A systematic review and meta-analysis. Cancer Epidemiol Biomarkers Prev 23: 1204-1212, 2014.

9. Templeton AJ, McNamara MG, Šeruga B, Vera-Badillo FE, Aneja P, Ocaña A, Leibowitz-Amit R, Sonpavde G, Knox JJ, Tran B, et al: Prognostic role of neutrophil-to-lymphocyte ratio in solid tumors: A systematic review and meta-analysis. J Natl Cancer Inst 106: dju124, 2014.

10. Orditura M, Galizia G, Diana A, Saccone C, Cobellis L, Ventriglia J, Iovino F, Romano C, Morgillo F, Mosca L, et al: Neutrophil to lymphocyte ratio (NLR) for prediction of distant metastasis-free survival (DMFS) in early breast cancer: A propensity score-matched analysis. ESMO Open 1: e000038, 2016.

11. Wei B, Yao M, Xing C, Wang W, Yao J, Hong Y, Liu Y and Fu P: The neutrophil lymphocyte ratio is associated with breast cancer prognosis: An updated systematic review and meta-analysis. Onco Targets Ther 9: 5567-5575, 2016.

12. Tang L, Li X, Wang B, Luo G, Gu L, Chen L, Liu K, Gao Y and Zhang X: Prognostic Value of neutrophil-to-lymphocyte ratio in localized and advanced prostate cancer: A systematic review and meta-analysis. PLoS One 11: e0153981, 2016.

13. Xue P, Kanai M, Mori Y, Nishimura T, Uza N, Kodama Y, Kawaguchi Y, Takaori K, Matsumoto S, Uemoto S and Chiba T: Neutrophil-to-lymphocyte ratio for predicting palliative chemotherapy outcomes in advanced pancreatic cancer patients. Cancer Med 3: 406-415, 2014.

14. Cho IR, Park JC, Park CH, Jo JH, Lee HJ, Kim S, Shim CN, Lee H, Shin SK, Lee SK and Lee YC: Pre-treatment neutrophil to lymphocyte ratio as a prognostic marker to predict chemotherapeutic response and survival outcomes in metastatic advanced gastric cancer. Gastric Cancer 17: 703-710, 2014.

15. Nakamura K, Nagasaka T, Nishida T, Haruma T, Ogawa C, Kusumoto T, Seki N and Hiramatsu Y: Neutrophil to lymphocyte ratio in the pre-treatment phase of final-line chemotherapy predicts the outcome of patients with recurrent ovarian cancer. Oncol Lett 11: 3975-3981, 2016.

16. Balkwill $\mathrm{F}$ and Mantovani A: Inflammation and cancer: Back to Virchow? Lancet 357: 539-545, 2001.

17. Menon S, Shin S and Dy G: Advances in cancer immunotherapy in solid tumors. Cancers (Basel) 8: 106, 2016.

18. Kusumanto YH, Dam WA, Hospers GA, Meijer C and Mulder NH: Platelets and granulocytes, in particular the neutrophils, form important compartments for circulating vascular endothelial growth factor. Angiogenesis 6: 283-287, 2003.

19. Hagerling C, Casbon AJ and Werb Z: Balancing the innate immune system in tumor development. Trends Cell Biol 25: 214-220, 2015.

20. Murray PJ, Allen JE, Biswas SK, Fisher EA, Gilroy DW, Goerdt S, Gordon S, Hamilton JA, Ivashkiv LB, Lawrence T, et al: Macrophage activation and polarization: Nomenclature and experimental guidelines. Immunity 41: 14-20, 2014.

21. Pillay J, Tak T, Kamp VM and Koenderman L: Immune suppression by neutrophils and granulocytic myeloid-derived suppressor cells: Similarities and differences. Cell Mol Life Sci 70: 3813-3827, 2013.

22. Walker JA, Barlow JL and McKenzie AN: Innate lymphoid cells-how did we miss them? Nat Rev Immunol 13: 75-87, 2013.

23. Hanahan D and Weinberg RA: The hallmarks of cancer. Cell 100: 57-70, 2000.

24. Lin EY and Pollard JW: Role of infiltrated leucocytes in tumour growth and spread. Br J Cancer 90: 2053-2058, 2004. 
25. Güngör N, Knaapen AM, Munnia A, Peluso M, Haenen GR, Chiu RK, Godschalk RW and van Schooten FJ: Genotoxic effects of neutrophils and hypochlorous acid. Mutagenesis 25: 149-154, 2010.

26. Nicolás-Ávila J, Adrover JM and Hidalgo A: Neutrophils in homeostasis, immunity, and cancer. Immunity 46: 15-28, 2017.

27. Uribe-Querol E and Rosales C: Neutrophils in cancer: Two sides of the same coin. J Immunol Res 2015: 983698, 2015.

28. Grivennikov SI, Greten FR and Karin M: Immunity, inflammation, and cancer. Cell 140: 883-899, 2010.

29. Raungkaewmanee S, Tangjitgamol S, Manusirivithaya S, Srijaipracharoen S and Thavaramara T: Platelet to lymphocyte ratio as a prognostic factor for epithelial ovarian cancer. J Gynecol Oncol 23: 265-273, 2012.

30. Liu R, Zheng S, Yuan Q, Zhu P, Li B, Lin Q, Shi W, Min Y, Ge Q and Shao Y: The prognostic significance of combined pretreatment fibrinogen and neutrophil-lymphocyte ratio in various cancers: A systematic review and meta-analysis. Dis Markers 2020: 4565379, 2020

31. Peng SM, Yu N, Ren JJ, Xu JY, Chen GC, Yang JR, Li ZN, Du HZ, Li DP, Zhang YS and Qin LQ: The Geriatric Nutritional Risk Index as a prognostic factor in patients with advanced non-small-cell lung cancer. Nutr Cancer: Dec 24, 2020 (Epub ahead of print). doi: 10.1080/01635581.2020.1865423.
32. Luo X, Yu B, Jiang N, Du Q, Ye X, Li H, Wang WQ and Zhai Q: Chemotherapy-induced reduction of neutrophil-to-lymphocyte ratio is associated with better survival in pancreatic adenocarcinoma: A meta-analysis. Cancer Control 27: 1073274820977135 , 2020.

33. Nogueira-Costa G, Fernandes I, Gameiro R, Gramaça J, Xavier AT and Pina I: Prognostic utility of neutrophil-to-lymphocyte ratio in patients with metastatic colorectal cancer treated using different modalities. Curr Oncol 27: 237-243, 2020.

34. Iwai N, Okuda T, Sakagami J, Harada T, Ohara T, Taniguchi M, Sakai H, Oka K, Hara T, Tsuji T, et al: Neutrophil to lymphocyte ratio predicts prognosis in unresectable pancreatic cancer. Sci Rep 10: 18758, 2020.

35. Vernieri C, Mennitto A, Prisciandaro M, Huber V, Milano M, Rinaldi L, Cona MS, Maggi C, Ferrari B, Manoukian S, et al: The neutrophil-to-lymphocyte and platelet-to-lymphocyte ratios predict efficacy of platinum-based chemotherapy in patients with metastatic triple negative breast cancer. Sci Rep 8: 8703, 2018.

(i)(9) This work is licensed under a Creative Commons International (CC BY-NC-ND 4.0) License. 